\title{
Synthesis and biological evaluation of quinoxaline di- $N$-oxide derivatives with in vitro trypanocidal activity
}

\author{
Silvia Pérez-Silanes ${ }^{\text {a,b }}$, Enrique Torres ${ }^{a}$, Leire Arbillaga ${ }^{c}$, Javier Varela $^{\text {d, }}$, Hugo \\ Cerecetto $^{d}$, Mercedes González ${ }^{d}$, Amaya Azqueta ${ }^{\text {c,e }}$, Elsa Moreno-Viguri ${ }^{a, b^{*}}$ \\ a Department of Organic and Pharmaceutical Chemistry. University of Navarra. \\ Irunlarrea s/n, 31008 Pamplona, Spain. \\ ${ }^{\mathrm{b}}$ Institute of Tropical Health University of Navarra. Irunlarrea s/n, 31008 Pamplona, \\ Spain. \\ ${ }^{c}$ Department of Pharmacology and Toxicology. University of Navarra. Irunlarrea $\mathrm{s} / \mathrm{n}$, \\ 31008 Pamplona, Spain.

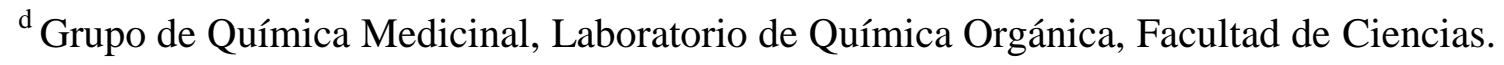 \\ Universidad de la República. Iguá 4225, 11400 Montevideo, Uruguay. \\ ${ }^{\mathrm{e}}$ IdiSNA, Navarra Institute for Health Research. Recinto de Complejo Hospitalario de \\ Navarra c/Irunlarrea 3 Pamplona 31008
}

\begin{abstract}
We report the synthesis and in vitro activity against T. cruzi epimastigotes of 15 novel quinoxaline derivatives. Ten of the derivatives presented $\mathrm{IC}_{50}$ values lower than the reference drugs $\mathrm{Nfx}$ and Bzn; four of them standed out with $\mathrm{IC}_{50}$ values lower than $1.5 \mu \mathrm{M}$. Moreover, unspecific cytotoxicity and genotoxicity studies are also reported. Compound 14 showed a SI higher than 24, whereas compound 10 was the only one that was negative in the genotoxicity screening.
\end{abstract}

Keywords: Chagas disease, Trypanosoma cruzi, Quinoxaline $N$-oxide, cytotoxicity, genotoxicity.

Abbreviations: BFX, benzofuroxan; CD, Chagas Disease; Nfx, Nifurtimox; Bnz, Benznidazol; NCE, New Chemical Entity; N.T., Not Tested; NTD, Neglected Tropical Diseases; PGI, percentage of growth inhibition; SI, Selectivity Index; TPP, Target Product Profile.

*Corresponding author: Dr. Elsa Moreno-Viguri. Institute of Tropical Health University of Navarra. E31008 Pamplona. Spain. e-mail: emviguri@unav.es 
Chagas disease (CD) is classified as one of the 17 Neglected Tropical Diseases (NTD) as defined by WHO. ${ }^{1}$ The 17 NTD account for a disease burden of at least 26 million disability-adjusted life years (DALYs) according to the Third WHO Report on Neglected Tropical Diseases ${ }^{2}$ and are under the Sustainable Development Goals SDG3. However, only the $0.6 \%$ of the new therapeutic products registered from 2000 to 2011 were indicated for NTD and none of them were a NCE. ${ }^{3}$ These facts highlight the urgent need of new effective and safe drugs for fighting NTDs.

$\mathrm{CD}$, also known as American trypanosomiasis, is caused by the protozoan parasite Trypanosoma cruzi. It used to be considered as a zoonotic disease affecting rural areas in low-middle income countries in Latin America. Nevertheless, human migration has spread out the disease worldwide and, according to WHO, 8 million people are currently infected. ${ }^{4}$ It is estimated that over 10000 people die every year from CD, and more than 25 million people are at risk of infection.

$\mathrm{CD}$ is curable if treatment is initiated soon after infection; therefore, access to diagnosis is essential. If not treated, $30 \%$ of the affected people develop heart damage and $10 \%$ suffer from digestive and/or neurological alterations. Nifurtimox (Lampit®) and Benznidazol (Rochagan ${ }^{\circledR}$ ) are the only available drugs for CD and they were developed more than 40 years ago. Neither of them is approved by the FDA. ${ }^{5}$ The major limitation of currently available drugs is their lower antiparasitic activity in the established chronic form of the disease, which is the most prevalent presentation. On the other hand, both drugs have undesired side effects that can lead to treatment discontinuation, which for Nfx include anorexia, nausea and vomiting causing severe weight loss, insomnia and irritability, while for Bnz the most common adverse effect is urticarial dermatitis. ${ }^{6,7}$ Three clinical trials have recently been conducted. A phase II proof-of-activity study of oral posaconazole in the treatment of asymptomatic chronic CD was completed in January 2015 and no conclusions have been reported. ${ }^{8}$ The phase II clinical trial for the treatment of chronic CD with posaconazole and benznidazole (CHAGASAZOL) and a higher treatment failure was observed in patients in the posaconazole groups than in the Bnz group. ${ }^{9,} 10$ Finally, a proof-of-concept study of the promising NCE E1224 was recently completed but the development of E1224 as monotherapy has been stopped and it will be considered for new combinatory regimens. ${ }^{5,11,12}$

This background justifies the urgent need for novel and better drugs to treat both acute and chronic phases. Quinoxaline derivatives are a chemical scaffold that has showed a wide spectrum of biological activities. ${ }^{13}$ Our group has vast experience in the synthesis 
and biological evaluation of quinoxaline derivatives with anti-cancer, antimycobacterium and anti-inflammatory activities among others. ${ }^{14-26}$ One of the projects has been focused on the study of novel quinoxaline derivatives as anti T. cruzi agents. In this field, over 200 derivatives have been prepared and evaluated as anti-trypanosomatid agents and some structural requirements have been established for their anti-chagasic in vitro activity. $20,25,27,28$ Therefore, it can be considered that the main structural requirements for the trypanocidal activity of quinoxaline derivatives are: the presence of the $\mathrm{N}$-oxide moiety and the insertion of electro withdrawing substituents on the quinoxaline ring (e.g. fluoro, chloro, trifluoromethyl...) Despite the general opinion about the toxicity associated with $\mathrm{N}$-oxides, it has been reported that quinoxaline derivatives mutagenicity seems to be associated with the substituents on the heterocycle. ${ }^{29}$ With this background and with the aim of identifying a new lead with higher potency and selectivity and a better drug target profile a series of 15 novel quinoxaline derivatives were designed considering the structural requirements previously established by our group and the insertion of alicyclic amines of biological interest. Piperazine derivatives have been explored for their interest as antitrypanosomatid agents and their activity as inhibitors of different targets of interest to fight against $\mathrm{CD}$ has been reported. ${ }^{30-32}$ Structural similarity can be observed between the proposed compounds and fluoroquinolones (Figure 1), a family of well-known antibacterial agents that have shown interesting activity data against trypanosomatids. ${ }^{33-}$ 35

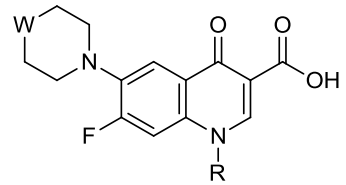

A

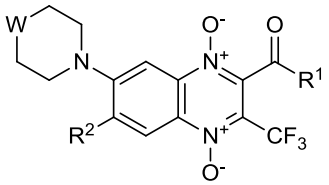

B

Figure 1. Structural similarity between fluoroquinolones (A) and proposed quinoxaline derivatives (B).

The design, synthesis and in vitro evaluation of new quinoxaline derivatives (1-15) as antitrypanosomal agents are described and SARs are discussed. The non-specific toxicity against mammalian cells was studied in order to evaluate the quinoxaline selectivity to the parasites and the SOS/umu test was included as a preliminary genotoxicity screening assay.

The designed compounds (1-15) were synthesized according to the sequence of reactions outlined in Scheme 1. 


$$
\text { (n) }
$$

BFX<smiles></smiles>
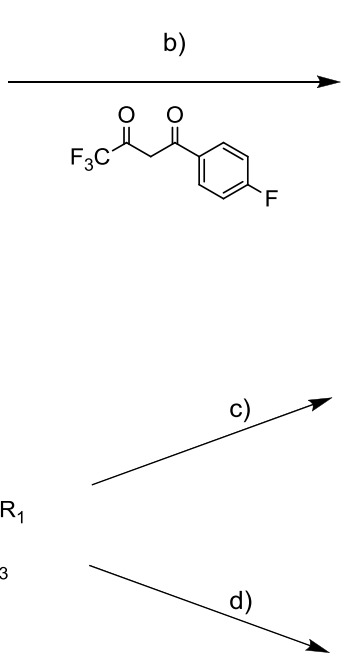
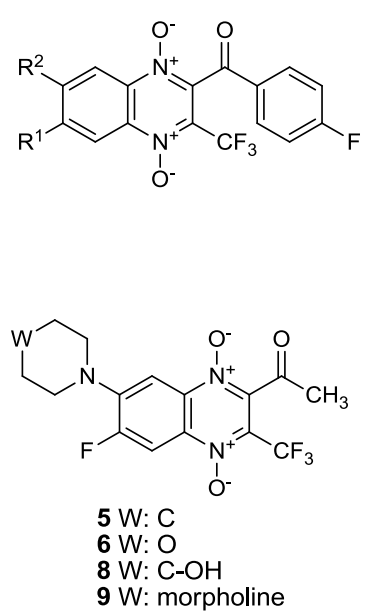<smiles></smiles>

$10 \mathrm{~W}: \mathrm{C}$ $11 \mathrm{~W}: \mathrm{C}-\mathrm{OH}$
$1 R^{1} / R^{2}: H / C$

$2 R^{1} / R^{2}: C l / C$

$3 \mathrm{R}^{1} / \mathrm{R}^{2}: \mathrm{H} / \mathrm{F}$

$4 \mathrm{R}^{1} / \mathrm{R}^{2}: \mathrm{F} / \mathrm{F}$<smiles>[R]C(=O)c1c(C(F)(F)F)[n+]([O-])c2cc([R])c([R])cc2[n+]1[O-]</smiles>

$7 R^{1}: \mathrm{COCH}_{3} ; R^{2}: F ; R^{3}$ : thiomorpholine; $n: 1$ $12 \mathrm{R}^{1}: \mathrm{COCH}\left(\mathrm{CH}_{3}\right)_{2} ; \mathrm{R}^{2}: \mathrm{N}_{(}\left(\mathrm{CH}_{3}\right)_{2} ; \mathrm{R}^{3}:$ morpholine; n: 1 $13 \mathrm{R}^{1}: \mathrm{COCH}\left(\mathrm{CH}_{3}\right)_{2} ; \mathrm{R}^{2}: \mathrm{N}\left(\mathrm{CH}_{3}\right)_{2} ; \mathrm{R}^{3}$ : morpholine; n: 1 $14 \mathrm{R}^{1}$ : CO-2-furyl. $\mathrm{R}^{2}$ : $\mathrm{Cl}$; $\mathrm{R}^{3}$ : morpholine; $\mathrm{n}: 0$ $15 \mathrm{R}^{1}$ : $\mathrm{COOCH}_{2} \mathrm{CH}_{3} ; \mathrm{R}^{2}$ : $\mathrm{Cl} ; \mathrm{R}^{3}$ : morpholine; $\mathrm{n}: 0$

Scheme 1. Synthesis of quinoxaline derivatives 1-15.

a) Previously reported in ${ }^{27,36}$; b) toluene, triethylamine, MW; c) acetonitrile, triethylamine, r.t.; d) $N, N$ DMF, reflux.

4 new quinoxaline 1,4 -di- $N$-oxide derivatives (1-4) were prepared using a variation of the Beirut reaction using microwave irradiation in which the corresponding BFX reacted with the 1-(4-fluorophenyl)-4,4,4-trifluoro-1,3-butanedione using toluene as solvent and triethylamine as base. The use of microwave assisted organic synthesis reduced the reaction times and simplifies the purification of quinoxaline derivatives. This fact led to an increase in the yield mainly when quinoxalines are substituted by halogens in positions 6 and/or 7 of the ring.

Compounds substituted by a cyclic amine on positions 6 and/or 7 of the quinoxaline ring were prepared by nucleophilic aromatic substitution of the corresponding quinoxaline. All the quinoxalines presented a chloro or fluoro substituted on positions 6 and/or 7 of the ring as leaving group and different cyclic amines were used as nucleophiles. As expected, the fluoro acts as a better leaving group decreasing the reaction times in comparison with the chloro. The longer reaction times needed when a chloro was substituted on positions 6 and 7 of the heterocycle led to the generation of the mono-reduced quinoxaline (14 and 15) complicating the purification of the desired compounds. ${ }^{37,38}$ Different synthetic methodologies were used with the aim of preparing the desired compounds. When the reaction was carried out in $N, N-\mathrm{DMF}$, the fluoro 
could be replaced not only by the cyclic amine but also by the solvent (compound 12) making more difficult the isolation of the desired compound. Moreover, the use of reflux would facilitate the generation of $1-\mathrm{N}$-oxide quinoxaline derivatives (14 and 15). $N, N$-DMF was substituted by acetonitrile that could be used as aprotic polar solvent that would avoid the aforementioned difficulties. ${ }^{39-41}$ Finally, triethylamine was used as base and 6 compounds were prepared using these conditions $(5,6,8-11)$. All of the compounds were fully characterized by infrared spectroscopy, nuclear magnetic resonance and elemental microanalyses (See Supplementary material).

Compounds were assayed against the epimastigote form of $T$. cruzi, Tulahuen 2 strain in order to determine the percentage of growth inhibition (PGI) at $25 \mu \mathrm{M}$ and the $\mathrm{IC}_{50}$. Compounds showing $\mathrm{IC}_{50}$ lower than Nifurtimox $\left(\mathrm{IC}_{50}=7.7 \mu \mathrm{M}\right)$ are considered active and would be considered for further testing. ${ }^{42}$ Five compounds were selected and their unspecific cytotoxicity and genotoxicity were studied before moving on to advanced studies. Cytotoxicity was assayed in Vero cells and the $\mathrm{IC}_{50}$ was determined from the dose-response curve. The Selectivity Index (SI) is expressed as the ratio of $\mathrm{IC}_{50}$ in mammalian cells to $\mathrm{IC}_{50}$ in epimastigote. The mutagenic capacity of the selected compounds was studied using the SOS/umu screening test.

The primary screening data for all compounds at $25 \mu \mathrm{M}$ are shown in Table 1. The results demonstrate that 13 out of the 15 evaluated compounds presented PGI higher than $80 \%$. Moreover, 10 of the derivatives showed $\mathrm{IC}_{50}$ values lower than the reference drugs Nfx and Bzn, presenting 4 of them $\mathrm{IC}_{50}$ values lower than $1.5 \mu \mathrm{M}$.

Analysis of the activity data presented in Table 1 reveals that substitution of a halogen atom, on position 7 of the quinoxaline, by a cyclic amine led to a decrease of the in vitro anti-T. cruzi activity. This behavior can be observed when comparing compounds 5-9 with their parent compound $\mathbf{A}$ or, in general terms, comparing with compounds 1-4.

If considering mono or di-substitution by cyclic amines no conclusion can be achieved. Di-substituted derivatives 11 and 13 showed lower PGI and higher $\mathrm{IC}_{50}$ than their mono-substituted analogues 8 and 12. Meanwhile, derivative 10 presented very interesting data, even better than its mono-substituted analogue $\mathbf{5}$.

Regarding the type of amine substituted on position 6 and/or 7, derivatives that present a piperidine on these positions (compounds $\mathbf{5}$ and 10) stand out with $\mathrm{IC}_{50}(2.6$ and 2.3 $\mu \mathrm{M}$, respectively) much better than the reference drugs. When piperidine is substituted by its bioisosteres morpholine or tiomorpholine or a hydroxyl moiety is substituted on 
position 6, the in vitro activity of these derivatives decreases (compound 5 vs. 6-8). It can be confirmed that the most decisive structural requirement, in terms of in vitro biological activity, is the presence of electron withdrawing substituents on positions 6 and/or 7. On the other hand, substitution on position 2 of the quinoxaline ring seems not to play such an important role on biological activity as previously observed. ${ }^{27,}{ }^{36}$ This behavior can also be observed in compounds 1-4 that present the best activity data.

Table 1. In vitro trypanosomicidal activity of quinoxaline derivatives.

\begin{tabular}{l|lll|ll}
\hline & \multicolumn{3}{|c|}{} & \multicolumn{2}{|l}{ T. cruzi epimastigote } \\
Tulahuen 2
\end{tabular}

[a] Previously reported in ${ }^{27}$. [b] Percentage of growth inhibition of epimastigote growth of Tulahen 2 strain, dose $=25 \mu \mathrm{M}$. [c] Concentration (in $\mu \mathrm{M}$ ) that inhibits $50 \%$ of epimastigote form of $T$. cruzi growth. The results are the mean of three independent experiments with an SD less than $10 \%$ in all cases. [d] quinoxaline- $1-N$-oxide

Throughout the activity optimization process we were mindful of the importance of a good toxicological profile that would guarantee not only a potent compound but also a safe drug. Therefore and owing to the interesting in vitro trypanosomicidal activity 
showed by the quinoxaline derivatives, 5 compounds (3-5, 10 and 14) were selected considering their $\mathrm{IC}_{50}$ value (compounds $\mathbf{3 , 4}$ and 5) and from a structural point of view compound $\mathbf{1 0}$ was included due to its significant activity and in order to study the influence of the substitution of an halogen by an amine. Compound $\mathbf{1 4}$ was selected to evaluate the influence of the $\mathrm{N}$-oxide on the toxicological profile of these derivatives. The in vitro cytotoxicity was studied using the tetrazolium-based colorimetric assay (MTT assay) in Vero cells. The $\mathrm{IC}_{50}$ values after $48 \mathrm{~h}$ of incubation are shown in Table 2. The selective indexes (SI) were calculated as the ratio of $\mathrm{IC}_{50}$ in Vero cells to $\mathrm{IC}_{50}$ in T. cruzi Tulahuen 2 strain.

Moreover, the compounds were tested in the SOS/umu screening test for their genotoxicity capacity. The results obtained from the SOS/umu screening test for the selected compounds $(3-5,10,14)$ with and without metabolic activation are shown in Table 2. The evaluated doses of each compound were determined in previous toxicity studies on the test system.

Table 2. In vitro toxicological profile of quinoxaline derivatives.

\begin{tabular}{c|cc|cc|cc}
\hline & \multicolumn{2}{|c|}{$\begin{array}{c}\text { T.cruzi } \\
\text { epimastigote } \\
\text { Tulahuen 2 }\end{array}$} & \multicolumn{2}{c|}{ MTT Vero Cells } & \multicolumn{2}{c}{ SOS/umu } \\
& PGI (\%) & $\begin{array}{c}\mathbf{I C}_{50} \\
(\mu \mathbf{M})\end{array}$ & $\begin{array}{c}\mathbf{I C}_{50} \\
(\boldsymbol{\mu M})\end{array}$ & SI & -S9 & + +S9 \\
\hline $\mathbf{3}$ & 95.6 & 1.1 & 4.4 & 4.0 & + & + \\
$\mathbf{4}$ & 91.8 & 0.6 & 3.0 & 5.0 & + & + \\
$\mathbf{5}$ & 96.9 & 2.6 & 15.2 & 5.8 & + & + \\
$\mathbf{1 0}$ & 100 & 2.3 & 4.5 & 1.7 & - & - \\
$\mathbf{1 4}$ & 91.7 & 12.1 & 454.7 & 37.6 & + & + \\
\hline
\end{tabular}

Regarding the unspecific cytotoxicity assay, 4 compounds (3-5 and 10) presented SI lower than 10. Compound $\mathbf{1 4}$ was the only one that presented a SI value higher than 25 and could be considered moderately selective. This increase in the SI is mainly due to the low non specific cytotoxicity presented by this compound which does not present a $\mathrm{N}$-oxide moiety, generally related with toxic effects, in one of the nitrogens of the quinoxaline heterocycle.

The SOS/umu screening test revealed that 4 compounds (3-5 and 14) were genotoxic with and without metabolic activation and compound $\mathbf{1 0}$ was not genotoxic in any of the tested conditions. Interestingly, the absence of a fluoro substituent in the quinoxaline system was the only condition to loss the mutagenic capacity. From the genotoxicity point of view, this could point out the quinoxaline $\mathbf{1 0}$ for further studies. 
According to the SI values showed in Table 2 compound $\mathbf{1 4}$ could be considered for further testing so that its activity against the trypomastigote and amastigote forms of $T$. cruzi should be explored. However, compound 14 was genotoxic in both tested conditions, with and without metabolic activation. Therefore, none of the tested compounds will continue for further development.

In conclusion, thirteen new 1,4-di- $N$-oxide and two 1- $N$-oxide quinoxaline derivatives were synthesized and evaluated against $T$. cruzi identifying new derivatives with potent in vitro anti-T. cruzi activity. Ten out of the fifteen evaluated compounds resulted more active than the reference drugs $\mathrm{Nfx}$ and Bnz. Four of them stand out with $\mathrm{IC}_{50}$ lower than $1.5 \mu \mathrm{M}$. Due to the toxicity issues usually associated to this family of compounds; unspecific cytotoxicity assay and a genotoxicity screening test were included in the very early stages of the project. Four out of the five tested compounds were mutagenic in both tested conditions. Compound $\mathbf{1 0}$ was not mutagenic; however, its low SI value ( $\mathrm{SI}=1.7$ ) led us to discontinue the biological studies of this family of compounds against T. cruzi. An appropriate toxicological profile study should consider not only unspecific cytotoxicity as usual but also mutagenicity issues. Including toxicological studies in the very early phases of biological evaluation would allow reducing costs and time in the overall drug discovery process.

\section{Acknowledgements}

The authors are grateful to Ms. Celia Goñi, Ms. Carmen Elizalde and Mr. Gorka Ruiz for their kind help. This work has been carried out with the financial support of FIMA (Fundación para la Investigación Médica Aplicada) from the University of Navarra and Fundación Caja Navarra (Project n. 70314) and CSIC- Uruguay (Project n. 612). This work was done in the auspices of RIDIMEDCHAG Network- CYTED. ET is indebted to the University of Navarra for a grant. AA thanks the Ministerio de Economía y Competitividad ('Ramón y Cajal' programme, 2013) of the Spanish Government for personal support.

\section{References}

1. http://www.who.int/neglected_diseases/diseases/en/ (04/10/2015).

2. Investing to overcome the global impact of neglected tropical diseases. Third WHO report on neglected tropical diseases. 2015.

3. Martin-Plaza, J.; Chatelain, E. Novel therapeutic approaches for neglected infectious diseases. J. Biomol. Screen. 2015, 20, 3.

4. Chagas disease (American trypanosomiasis). Fact sheet $\mathrm{N}^{\circ} 340.2015$.

5. Neitz, R. J.; Chen, S.; Supek, F.; Yeh, V.; Kellar, D.; Gut, J.; Bryant, C.; Gallardo-Godoy, A.; Molteni, V.; Roach, S. L.; Chatterjee, A. K.; Robertson, S.; 
Renslo, A. R.; Arkin, M.; Glynne, R.; McKerrow, J.; Siqueira-Neto, J. L. Lead identification to clinical candidate selection: drugs for Chagas disease. J. Biomol. Screen. 2015, 20, 101.

6. Bern, C. Antitrypanosomal therapy for chronic Chagas' disease. N. Eng. J. Med. 2011, 364 .

7. Outlook: Chagas Disease. Nature. 2010, 465, S3.

8. A Study of the Use of Oral Posaconazole (POS) in the Treatment of Asymptomatic Chronic Chagas Disease (P05267) (STOP CHAGAS). NCT01377480. www.clinicaltrials.gov $(04 / 28 / 2015)$.

9. Molina, I.; Gomez i Prat, J.; Salvador, F.; Trevino, B.; Sulleiro, E.; Serre, N.; Pou, D.; Roure, S.; Cabezos, J.; Valerio, L.; Blanco-Grau, A.; Sanchez-Montalva, A.; Vidal, X.; Pahissa, A. Randomized trial of posaconazole and benznidazole for chronic Chagas'disease. N. Eng. J. Med. 2014, 370, 1899.

10. Clinical trial for the treatment of chronic Chagas disease with posaconazole and benznidazole (CHAGASAZOL). NCT01162967. www.clinicaltrials.gov (04/28/2015).

11. Drugs for Neglected Diseases Initiative. http://www.dndi.org/ (04/28/2015).

12. Proof-of-Concept Study of E1224 to Treat Adult Patients With Chagas Disease. NCT01489228. www.clinicaltrials.gov (04/28/2015).

13. Jampilek, J. Recent advances in design of potential quinoxaline anti-infectives. Curr. Med. Chem. 2014, 21, 4347.

14. Urquiola, C.; Vieites, M.; Aguirre, G.; Marin, A.; Solano, B.; Arrambide, G.; Noblia, P.; Lavaggi, M. L.; Torre, M. H.; Gonzalez, M.; Monge, A.; Gambino, D.; Cerecetto, H. Improving anti-trypanosomal activity of 3-aminoquinoxaline-2carbonitrile N1,N4-dioxide derivatives by complexation with vanadium. Bioorg. Med. Chem. 2006, 14, 5503.

15. Solano, B.; Junnotula, V.; Marin, A.; Villar, R.; Burguete, A.; Vicente, E.; Perez-Silanes, S.; Aldana, I.; Monge, A.; Dutta, S.; Sarkar, U.; Gates, K. S. Synthesis and biological evaluation of new 2-arylcarbonyl-3-trifluoromethylquinoxaline 1,4-di-Noxide derivatives and their reduced analogues. J. Med. Chem. 2007, 50, 5485.

16. Marin, A.; Moreira Lima, L.; Solano, B.; Vicente, E.; Perez Silanes, S.; Maurel, S.; Sauvain, M.; Aldana, I.; Monge, A.; Deharo, E. Antiplasmodial structure-activity relationship of 3-trifluoromethyl-2-arylcarbonylquinoxaline 1,4-di-N-oxide derivatives. Exp. Parasitol. 2008, 118, 25.

17. Urquiola, C.; Gambino, D.; Cabrera, M.; Lavaggi, M. L.; Cerecetto, H.; Gonzalez, M.; de Cerain, A. L.; Monge, A.; Costa-Filho, A. J.; Torre, M. H. New copper-based complexes with quinoxaline N1,N4-dioxide derivatives, potential antitumoral agents. J. Inorg. Biochem. 2008, 102, 119.

18. Vicente, E.; Villar, R.; Burguete, A.; Solano, B.; Perez-Silanes, S.; Aldana, I.; Maddry, J. A.; Lenaerts, A. J.; Franzblau, S. G.; Cho, S. H.; Monge, A.; Goldman, R. C. Efficacy of quinoxaline-2-carboxylate 1,4-di-N-oxide derivatives in experimental tuberculosis. Antimicrob. Agents Chemother. 2008, 52, 3321.

19. Villar, R.; Vicente, E.; Solano, B.; Perez-Silanes, S.; Aldana, I.; Maddry, J. A.; Lenaerts, A. J.; Franzblau, S. G.; Cho, S. H.; Monge, A.; Goldman, R. C. In vitro and in vivo antimycobacterial activities of ketone and amide derivatives of quinoxaline 1,4-diN-oxide. J. Antimicrob. Chemother. 2008, 62, 547.

20. Ancizu, S.; Moreno, E.; Torres, E.; Burguete, A.; Perez-Silanes, S.; Benitez, D.; Villar, R.; Solano, B.; Marin, A.; Aldana, I.; Cerecetto, H.; Gonzalez, M.; Monge, A. Heterocyclic-2-carboxylic acid (3-cyano-1,4-di-N-oxidequinoxalin-2-yl)amide derivatives as hits for the development of neglected disease drugs. Molecules. 2009, 14, 2256. 
21. Vicente, E.; Perez-Silanes, S.; Lima, L. M.; Ancizu, S.; Burguete, A.; Solano,

B.; Villar, R.; Aldana, I.; Monge, A. Selective activity against Mycobacteriumtuberculosis of new quinoxaline 1,4-di-N-oxides. Bioorg. Med. Chem. 2009, 17, 385.

22. Ancizu, S.; Moreno, E.; Solano, B.; Villar, R.; Burguete, A.; Torres, E.; PerezSilanes, S.; Aldana, I.; Monge, A. New 3-methylquinoxaline-2-carboxamide 1,4-di-Noxide derivatives as anti-Mycobacterium tuberculosis agents. Bioorg. Med. Chem. 2010, $18,2713$.

23. Moreno, E.; Ancizu, S.; Perez-Silanes, S.; Torres, E.; Aldana, I.; Monge, A. Synthesis and antimycobacterial activity of new quinoxaline-2-carboxamide 1,4-di-Noxide derivatives. Eur. J. Med. Chem. 2010, 45, 4418.

24. Burguete, A.; Pontiki, E.; Hadjipavlou-Litina, D.; Ancizu, S.; Villar, R.; Solano, B.; Moreno, E.; Torres, E.; Perez, S.; Aldana, I.; Monge, A. Synthesis and biological evaluation of new quinoxaline derivatives as antioxidant and anti-inflammatory agents. Chem. Biol. Drug Design. 2011, 77, 255.

25. Torres, E.; Moreno, E.; Ancizu, S.; Barea, C.; Galiano, S.; Aldana, I.; Monge, A.; Perez-Silanes, S. New 1,4-di-N-oxide-quinoxaline-2-ylmethylene isonicotinic acid hydrazide derivatives as anti-Mycobacterium tuberculosis agents. Bioorg. Med. Chem. Lett. 2011, 21, 3699.

26. Ancizu, S.; Castrillo, N.; Perez-Silanes, S.; Aldana, I.; Monge, A.; Delagrange, P.; Caignard, D. H.; Galiano, S. New quinoxaline derivatives as potential MT(1) and MT(2) receptor ligands. Molecules. 2012, 17, 7737.

27. Benitez, D.; Cabrera, M.; Hernandez, P.; Boiani, L.; Lavaggi, M. L.; Di Maio, R.; Yaluff, G.; Serna, E.; Torres, S.; Ferreira, M. E.; Vera de Bilbao, N.; Torres, E.; Perez-Silanes, S.; Solano, B.; Moreno, E.; Aldana, I.; Lopez de Cerain, A.; Cerecetto, H.; Gonzalez, M.; Monge, A. 3-Trifluoromethylquinoxaline N,N'-dioxides as antitrypanosomatid agents. Identification of optimal anti-T. cruzi agents and mechanism of action studies. J. Med. Chem. 2011, 54, 3624.

28. Moreno-Viguri, E.; Pérez-Silanes, S. Quinoxaline 1,4-di-N-oxide Derivatives: Interest in the Treatment of Chagas Disease. Rev. Virtual Química. 2013, 5, 1101.

29. Gabay, M.; Cabrera, M.; Maio, R. D.; Paez, J. A.; Campillo, N.; Lavaggi, M. L.; Cerecetto, H.; Gonzalez, M. Mutagenicity of N-oxide containing heterocycles and related compounds: experimental and theoretical studies. Curr. Top. Med. Chem. 2014, 14, 1374.

30. Bonnet, B.; Soullez, D.; Girault, S.; Maes, L.; Landry, V.; Davioud-Charvet, E.; Sergheraert, C. Trypanothione reductase inhibition/trypanocidal activity relationships in a 1,4-bis(3-aminopropyl)piperazine series. Bioorg. Med. Chem. 2000, 8, 95.

31. Jacobsen, W.; Christians, U.; Benet, L. Z. In vitro evaluation of the disposition of A novel cysteine protease inhibitor. Drug Metab Dispos. 2000, 28, 1343.

32. Papadopoulou, M. V.; Bloomer, W. D.; Rosenzweig, H. S.; Kaiser, M.; Chatelain, E.; Ioset, J. R. Novel 3-nitro-1H-1,2,4-triazole-based piperazines and 2amino-1,3-benzothiazoles as antichagasic agents. Bioorg. Med. Chem. 2013, 21, 6600.

33. Nenortas, E.; Burri, C.; Shapiro, T. A. Antitrypanosomal activity of fluoroquinolones. Antimicrob. Agents Chemother. 1999, 43, 2066.

34. Nenortas, E.; Kulikowicz, T.; Burri, C.; Shapiro, T. A. Antitrypanosomal activities of fluoroquinolones with pyrrolidinyl substitutions. Antimicrob. Agents Chemother. 2003, 47, 3015.

35. Ma, X.; Zhou, W.; Brun, R. Synthesis, in vitro antitrypanosomal and antibacterial activity of phenoxy, phenylthio or benzyloxy substituted quinolones. Bioorg. Med. Chem. Lett. 2009, 19, 986. 
36. Torres, E.; Moreno-Viguri, E.; Galiano, S.; Devarapally, G.; Crawford, P. W.; Azqueta, A.; Arbillaga, L.; Varela, J.; Birriel, E.; Di Maio, R.; Cerecetto, H.; Gonzalez, M.; Aldana, I.; Monge, A.; Perez-Silanes, S. Novel quinoxaline 1,4-di-N-oxide derivatives as new potential antichagasic agents. Eur. J. Med. Chem. 2013, 66, 324.

37. Charushin, V. N.; Mokrushina, G. A.; Tkachev, A. V. Nucleophilic substitutions in 6,7-difluoroquinoxalines. J. Fluorine Chem. 2001, 107, 71.

38. Chambers, R. D.; Martin, P. A.; Sandford, G.; Williams, D. L. H. Mechanisms of reactions of halogenated compounds: Part 7. Effects of fluorine and other groups as substituents on nucleophilic aromatic substitution. J. Fluorine Chem. 2008, 129, 998.

39. Dang, Z.; Yang, Y.; Ji, R.; Zhang, S. Synthesis and antibacterial activity of novel fluoroquinolones containing substituted piperidines. Bioorg. Med. Chem. Lett. 2007, 17, 4523.

40. Huang, X.; Zhang, A.; Chen, D.; Jia, Z.; Li, X. 4-Substituted 4-(1H-1,2,3-triazol1-yl)piperidine: novel $\mathrm{C} 7$ moieties of fluoroquinolones as antibacterial agents. Bioorg. Med. Chem. Lett. 2010, 20, 2859.

41. Parks, E. L.; Sandford, G.; Yufit, D. S.; Howard, J. A. K.; Christopher, J. A.; Miller, D. D. Synthesis of tetrahydropyrido- and pyrido-[1',2':1,2]imidazo[4,5b]pyrazine derivatives. J. Fluorine Chem. 2010, 131, 1086.

42. Romanha, A. J.; Castro, S. L.; Soeiro Mde, N.; Lannes-Vieira, J.; Ribeiro, I.; Talvani, A.; Bourdin, B.; Blum, B.; Olivieri, B.; Zani, C.; Spadafora, C.; Chiari, E.; Chatelain, E.; Chaves, G.; Calzada, J. E.; Bustamante, J. M.; Freitas-Junior, L. H.; Romero, L. I.; Bahia, M. T.; Lotrowska, M.; Soares, M.; Andrade, S. G.; Armstrong, T.; Degrave, W.; Andrade Zde, A. In vitro and in vivo experimental models for drug screening and development for Chagas disease. Mem. Inst. Oswaldo. Cruz. 2010, 105, 233. 


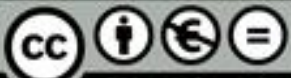

BY NC ND Esta obra está bajo una licencia de Creative Commons Reconocimiento-

NoComercial-SinObraDerivada 4.0 Internacional. 